\title{
Image Super-resolution Reconstruction Algorithm Based on Self- Similarity and Compressive Sensing
}

\author{
Luyang Yao and Kai Xie \\ School of Information Engineering, Beijing Institute of Graphic Communication, \\ Beijing 102600 \\ 513095174@qq.com,xiekai@bigc.edu.cn
}

\begin{abstract}
Traditional super-resolution reconstruction algorithm based on sparse representation can present a good visual effect, but it is time-consuming and largely dependents on the training databases. A new image super-resolution algorithm based on self-similarity and compressive sensing is proposed. Firstly, the input low resolution image is rotated to increase the diversity of the training databases for self-similarity learning and the medium-frequency $(M F)$ patches are exploited to predict more high-frequency (HF) details based on the self-similarity theory, instead of using the low-frequency (LF) patches. In this way, we can obtain the initial high-resolution image and regard it as the only training sample database. Then, sparse reconstruction process is carried out by adopting the K-SVD dictionary learning combined with the compressive sensing (CS) theory. Finally, to preserve sharper edges, the non-local means and iterative back projection algorithm are applied to optimize the reconstructed high-resolution image. During the process of search and match in self-similarity reconstruction, the Fast Library for Approximate Nearest Neighbors (FLANN) is used to replace the Approximate Nearest Neighbors (ANN). Experimental results validate the effectiveness of this method and show that the proposed algorithm can perform better both visually and quantitatively.
\end{abstract}

Keywords: super-resolution; self-similarity; patch rotation; $K$-SVD dictionary learning; compressive sensing; non-local means; iterative back projection

\section{Introduction}

Increasing the image resolution or super-resolution (SR) aims to enlarge the target image, meanwhile preserving the image quality as much as possible. However, the natural image captured by the imaging devices is prone to be of low resolution due to many factors: blur, camera shake, inadequate lighting, noise, etc. Therefore, how to enhance the resolution effectively by SR has become a hot topic for several years.

Super-resolution image reconstruction algorithms can be basically classified into three categories: the interpolation-based SR methods [1], the reconstruction-based SR methods [2-3] and example-based SR methods [4-13]. The interpolation-based SR methods are very simple and suitable for real-time applications, but they are of low reconstruction precision and poor restoration details. The image reconstruction-based SR methods apply a variety of prior knowledge to estimate the super-resolution image, which can obtain a sharper edge and reduce the zigzag effect than the interpolation-based SR methods. However, this method needs many reasonable prior assumptions and exists the registration problems between different image frames. Example-based SR approaches break the limitations existing in the traditional reconstruction-based algorithms. They learn the mapping relationship between the corresponding pre-processed low and high resolution training samples to recover the missed HF details, mainly including learningbased approach [4], neighborhood embedding approach [5] and sparse representation methods [6-18], etc. 
Yang [6-8] proposed the super-resolution algorithm based on the sparse representation under the CS theory. In order to obtain more additional information and enable the different types of image patches to be sparsely represented in the same sparse dictionary, it needs a large number of training samples and inevitably results in a long training time for sparse dictionary. The obtained dictionary usually lacks of effectiveness in practical applications. Zeyde [10] modified the algorithm of Yang, in which training data is constructed by the patches taken from the difference between high-resolution patches and the interpolated version of low-resolution patches. Besides, the use of K-SVD [11] dictionary learning algorithm shortens the computational time and obtains a better reconstruction result. Glasner [19] introduced the image self-similarity theory, using only a low-resolution image to reconstruct the high-resolution image. Yang [14] made some improvements combined with sparse representation theory on the basis of Glasner's work, which improves the reconstruction efficiency and quality. The reconstruction method in [17] modified the algorithm of Yang [6-8] with the CS theory, reducing the number of training dictionary but still using external several training images. Pan in [15-16] and Zhu in [18] modified the method in [17] not relying on any external images, assuming that the low resolution patches can be considered as the compressed sensing version of high resolution patches under the framework of CS theory.

Enlightened by the recently-proposed algorithms, an extended self-similarity SR method combined with the CS theory is proposed. In this paper, we don't use the interpolated version of low-resolution input image as the training sample for sparse representation, but the initial high-resolution image reconstructed by the self-similarity based on MF and HF patches. The proposed algorithm takes full advantage of the additional information implied in the image itself. Experimental results indicate that the proposed algorithm can not only reduce the training time of sparse dictionary, also enhance the quality of the reconstructed image both quantitatively and visually.

\section{Self-similarity Reconstruction Based on MF and HF Patches}

Glasner in [19] made an experiment with a large number of natural images, drawing the conclusion that: natural images tend to contain repetitive visual contents, whether they are in the same scale or across different scales.

Previous self-similarity SR reconstruction methods are almost all based on LF and HF, holding that there exists high correlation between LF and HF patches. However, the reference [20] has suggested that this assumption was content-specified and can't hold water for the natural images with complex textures. And then, it further showed that MF and $\mathrm{HF}$ patches were more relevant and easier to predict the missed high-frequency details.

The traditional example-based SR methods usually select a large number of training sample patches to guarantee the quality of the reconstructed image, which will increase the computational complexity undoubtedly. Besides, the predicted HF details are possibly not what we really need for the low resolution interpolated image. To solve these shortcomings, we rotate the low resolution input image to increase their diversities on the basis of multi-scale self-similarity. To ensure the diversities of training samples and not increase the computational complexity, the segmented patches are only rotated by $90^{\circ}$ and each patch is converted to a column vector. So the same content will have different expression forms. In this way, the obtained training sample patches are of diversity, which can more fully utilize the self-similarity between patches across the different scales.

In order to obtain more connections between the MF and HF patches that are of high relevance, first at all, we use Gaussian low-pass filter and down-sampling operator on the low resolution input image $I_{0}$, then generate the multi-scale down-sampled images $I_{-1}, \ldots \ldots$, $I_{-n}(n=2)$. The obtained Gaussian Pyramid is composed by $-n^{\text {th }}$ to $0^{\text {th }}$ layer (the largest scale layer, namely $I_{0}$ ). If we describe $i^{\text {th }}$ layer as $I_{\mathrm{i}}$, then it can be expressed as follows: 


$$
I_{i}=\left\{\begin{array}{lc}
I_{0} & i=0 \\
D\left(I_{i+1}\right) & -n \leq i<0
\end{array}\right.
$$

Where $D$ represents the smoothing (by Gaussian low-pass filter) and down-sampling operator. Then the corresponding $I_{\mathrm{i}}$ can be decomposed into LF component $L_{\mathrm{i}}$ and HF component $H_{\mathrm{i}}$ :

$$
\begin{array}{ll}
L_{i}=U\left(D\left(I_{i}\right)\right. & -n \leq i \leq 0 \\
H_{i}=I_{i}-L_{i} & -n \leq i \leq 0
\end{array}
$$

Where $U$ denotes the up-scale operator (by bicubic interpolation). The LF information in formula (2) can be further decomposed into LF and HF components. The LF component extracted from the $L_{\mathrm{i}}$ can be called MF information, which is described below:

$$
M_{i}=L_{i}-U\left(D\left(L_{i}\right)\right) \quad-n \leq i \leq 0
$$

After collecting the MF and HF information of the original input image $I_{0}$ and its subsampled images, we divide them into patches (5x5, overlapped by 3 pixels) and then obtain the MF and HF databases, which is shown in Figure 1.

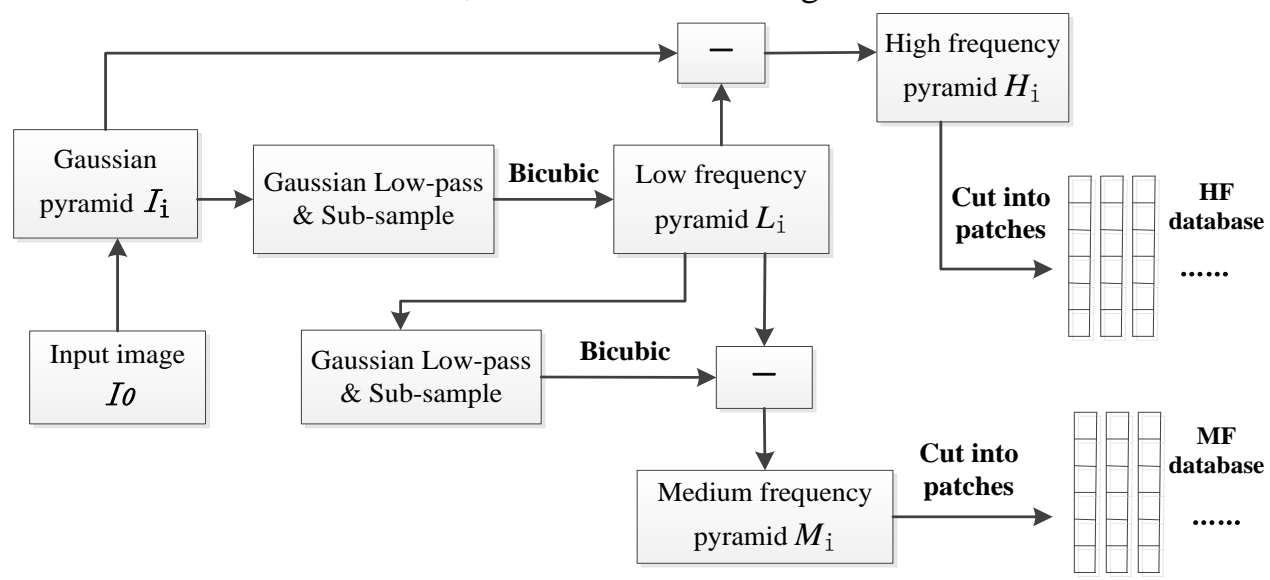

Figure 1. The Generation of the MF and HF Training Databases

We enlarge the low resolution image to the targeted size by bicubic interpolation noted as $L_{\mathrm{F}}$ and cut it into patches, as mentioned above. Then, for each patch of $L_{\mathrm{F}}$, we use the band-pass filter to obtain the corresponding MF information of $L_{\mathrm{F}}$, in order to search the most similar 9 MF patches by FLANN in the established MF database and find the corresponding HF patches in HF database. Finally, the Gaussian weighted average is used for the $9 \mathrm{HF}$ patches to generate the corresponding HF patch for low resolution patch $L_{\mathrm{F}}$, denoted as $H_{\mathrm{F}}$. So, the initial high-resolution image $H R_{0}$ can be expressed as:

$$
H R_{\mathrm{o}}=L_{F}+H_{F}
$$




\section{Super-resolution Reconstruction Based on CS Theory}

\subsection{Basic Theory of Image Sparse Representation}

Sparse representation based on the over-complete dictionary is a very effective image representation model, which can use a few non-zero elements in sparse coefficients to approximately represent the image.

According to the sparse representation theory, suppose an image patch $\left.y^{(\sqrt{n}} \times \sqrt{n}\right)$ has been converted into a column vector $y \in R^{n}$, then it can be represented as a sparse linear combination of atoms from an over-complete dictionary $D \in R^{n \times L}(n \leq L)$ :

$$
y=D \alpha
$$

Where $\alpha=\left[\alpha_{1}, \alpha_{2} \ldots . . \alpha_{L}\right]^{T} \in R^{L}$ denotes the sparse representation of the patch $y$ and most of the elements of it are zero. Solving the equation above is ill-posed and exists infinite solutions. In order to minimize the number of non-zero elements in sparse representation vector $\alpha$, the equation above can be transformed as :

$$
\min \|\alpha\|_{0} \quad \text { s.t. } \quad \mathrm{y}=D \alpha
$$

Unfortunately, due to the non-convexity of $L_{0}$ norm, to solve the formula (7) is a highly discrete problem. Besides, to find the most sparse coefficients of $\alpha$ from the overcomplete dictionary is a typical NP-hard problem. Therefore, we have to choose the suboptimal approximation algorithm to replace it. As early as 1999 , a study researched by Chen [22] found that the sparse solutions of $L_{0}$ norm can be converted into ones of $L_{1}$ norm when the signals are sparse enough. Hence, the formula (7) can be transformed into a combinatorial optimization problem as:

$$
\min \|\alpha\|_{1} \quad \text { s.t. }\|\mathrm{y}-D \alpha\|_{2} \leq \varepsilon
$$

Where $\varepsilon$ is the allowed root mean-square reconstruction error. From the formula above, it is noticeable that the selection of the sparse dictionary $D$ plays a crucial role for the unique solution of formula (8). In this paper, we choose the K-SVD [11] method to substitute the Feature-Sign Search (FSS) method of Yang [6-8] to improve the efficiency and quality of training dictionary.

\subsection{Self-similarity Super-Resolution Reconstruction Under CS Theory}

Compressive sensing theory suggests that the low-resolution image patches can be considered as the compressed sampling version of the high-resolution image patches. In mild conditions, the low-resolution image patches can be recovered correctly to highresolution image patches by the sparse representation theory. Besides, the low-resolution and high-resolution image patches can be approximately assumed to be of the same sparse coefficients. Due to the existence of high similarity between the low-resolution and highresolution patches, we use initial high-resolution image $H R_{0}$ as the training image for CS reconstruction.

In this paper, supposing the size of low-resolution patch to be reconstructed is $N x N$ and its corresponding column vector $I_{\text {low }}$ is $N^{2} x 1$. We can approximately hold that the lowresolution image can be obtained from the initial high-resolution image $H R_{0}$, for $H R_{0}$ contains lots of similar image patches and can be regarded as the corrupted version of the high-resolution image to be reconstructed. So, while the magnification factor is $K$, the size of corresponding high-resolution patch is $K N x K N$ and it can be converted into column vector $I_{\text {high }}$ of size $K^{2} N^{2} x 1$. Then, the relationship between the $I_{\text {low }}$ and $I_{\text {high }}$ can be described as below:

$$
I_{\text {low }}=B S \cdot I_{\text {high }}
$$


Where $B$ denotes the Gaussian low-pass filter and $S$ is the image down-sampling operator. From the formula (6) in the sparse representation theory, $I_{l o w}$ and $I_{\text {high }}$ can be represented linearly by over-complete dictionaries. That is:

$$
I_{\text {low }}=D_{\text {low }} \alpha=B S \cdot D_{\text {high }} \alpha
$$

In the formula above, $D_{h i g h}$ is trained from corresponding initial high-resolution image $H R_{0}$ by the K-SVD dictionary learning method.

According to the formula (8), the formula (10) can be modified into the following combinatorial optimization problem:

$$
\min \left\|\alpha_{0}\right\| \text { s.t. } I_{\text {low }}=B S \cdot D_{\text {high }} \alpha=D_{\text {low }} \alpha
$$

Using the equivalent replacement method, the relationship between $D_{\text {low }}$ and $D_{\text {high }}$ can be quickly described as:

$$
D_{\text {low }}=D_{\text {high }} B S
$$

Once the $D_{\text {low }}$ is obtained, we can exploit the Orthogonal Matching Pursuit (OMP) to solve the sparse coefficients $\alpha$. Then, the high-resolution image patch to be reconstructed can be expressed as follows:

$$
I_{\text {high }}=D_{\text {high }} \alpha
$$

After reconstructing the each high-resolution patch, we merge all the patches by pixel weighted method to generate the high-resolution image.

\subsection{Optimization Process}

In order to further improve the resolution of the reconstructed image, the non-local similarity [24] and the iterative back projection algorithm [25] are used to optimize the reconstruction results.

\subsubsection{Local Constraint Algorithm}

The core of the local constraint [24] is based on image patches to search similarity patches for each image patch. Once the similar patches are found, we can use the different weighted values to obtain the final patch, which relates the global similarity of image together and can make the final reconstructed image sharper and more precise.

For each image patch $(5 \times 5)$, we search from central pixel of each patch by the preset threshold value to calculate the Euclidean distance between the similar patches, which can be expressed below:

$$
d_{i}^{k}=\left\|x_{i}-x_{i}^{k}\right\| \leq \text { threshold }
$$

Where threshold represents the preset distance threshold, $x_{i}$ is the each patch to be

locally constrained, $x_{i}^{k}$ is the similar patches to be searched. In this experiment, we select eight most similar image patches for each patch, obtaining the final image patch by linear weighting, which is presented as follows:

$$
\begin{gathered}
x_{i}=\sum_{k=1}^{M} w_{i}^{k} x_{i}^{k}(M \leq 8) \\
w_{i}=\exp \left(-d_{i}^{k} / h\right) / c_{k} \\
c_{k}=\sum_{k=1}^{M} \exp \left(-d_{i}^{k} / h\right)
\end{gathered}
$$


Where ${ }^{w_{i}}$ is the weight coefficient and the ${ }^{c_{k}}$ represents weight normalization factor. $h$ controls the descending speed of weight value as the Euclidean distance increases.

\subsubsection{Iterative Back Projection Algorithm}

To ensure that the final reconstructed high resolution image can be consistent with the input low resolution image, we adopt the iterative back projection (IBP) to optimize the reconstructed image globally, which can effectively reduce the diffusion of errors. IBP algorithm can be illustrated as follows:

$$
X_{t+1}=X_{t}+\left(\left(Y-D X_{t}\right)_{\uparrow}\right) * \text { GuassLowFilter }
$$

Where $X_{\mathrm{t}+1}$ represents the $(t+1)^{\text {th }}$ iteration result of high-resolution image, $Y$ is the given input low-resolution image, $\uparrow^{\uparrow}$ is the interpolation operation and $D$ is the downsample operation and GuassLowFilter represents the Gaussian low-pass filter.

\subsection{The Procedure of Super-resolution based on CS theory and Self-similarity}

Now, we summarize the whole proposed algorithm mentioned above as follows and the general process as well as the flowchart of our proposed algorithm is illustrated in Figure 2.

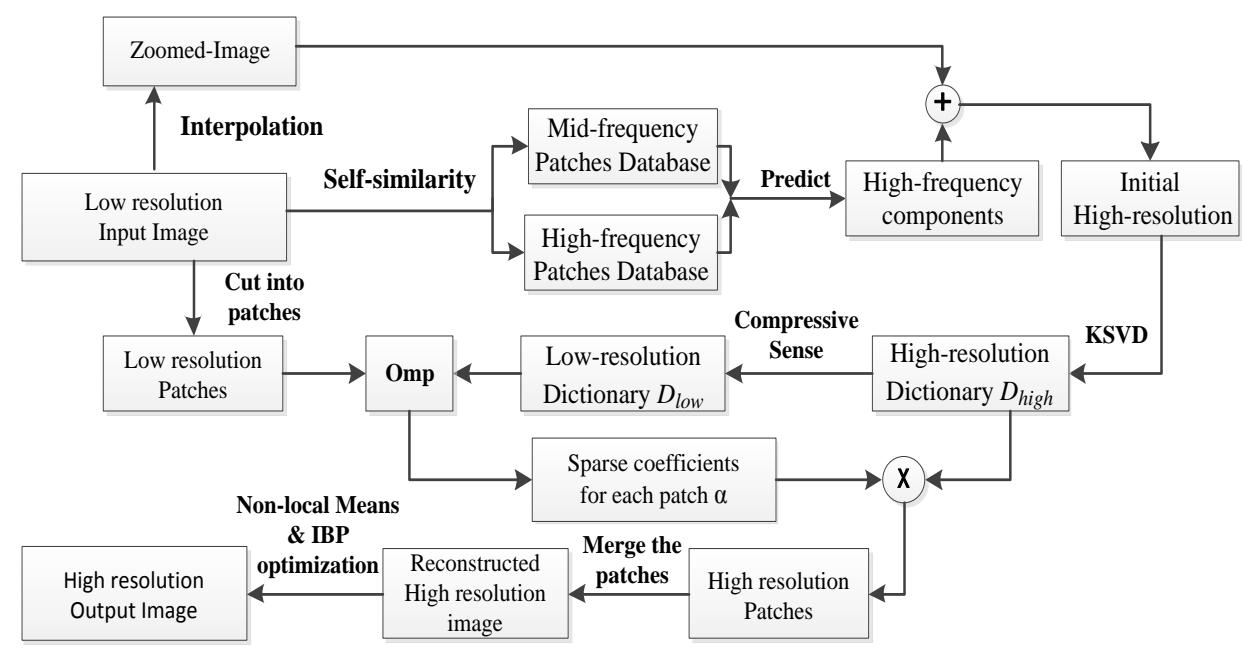

Figure 2. The General Process of the Proposed Algorithm

(1) Load the low-resolution input image $I_{0}$,rotate it and down-sample,then divide them into overlapped patches, generating the MF and $\mathrm{HF}$ training databases.

(2) Use the MF training patches to predict the missed HF information based on the principle of self-similarity, then obtain the initial high-resolution image $H R_{0}$.

(3) Divide the initial high-resolution image $H R_{0}$ into patches and exploit the K-SVD algorithm to implement the dictionary training, obtaining the high-resolution dictionary $D_{\text {high }}$.

(4) Generate the low-resolution dictionary $D_{\text {low }}$ according to the formula (12) by the CS theory.

(1) Divide the low-resolution image into patches and use OMP for low-resolution dictionary $D_{\text {low }}$ to obtain the each sparse coefficient $\alpha$ for each low-resolution image patch.

(2) Multiply the each sparse coefficient $\alpha$ by $D_{\text {high }}$ to obtain the corresponding highresolution image patch according to the formula (13). 
(3) Merge all the high-resolution image patches into high-resolution image, then optimize it by performing non-local means and iterative back projection algorithm.

\section{Experiment Analysis and Results}

Here, we conduct the SR experiment on eight low-resolution testing images (128x128) with the magnification factor 2 and 4 in Figure 3. These images cover many contents including humans, animals, and plants, which can validate the university of our proposed algorithm.

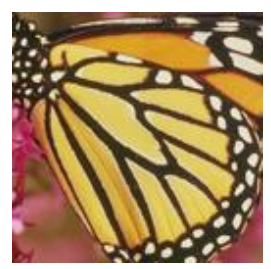

(a) Butterfly

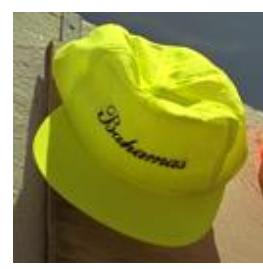

(b) Hat

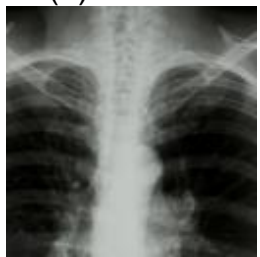

(d) Chest

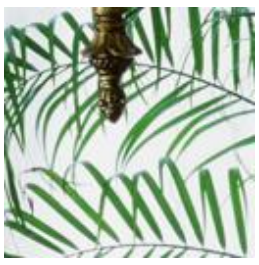

(c) Leaves

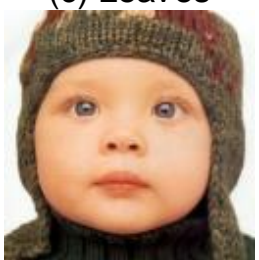

(e) Baby

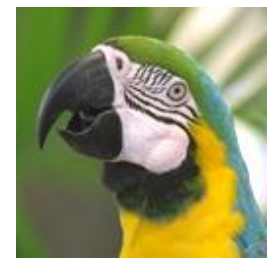

(d) Parrots

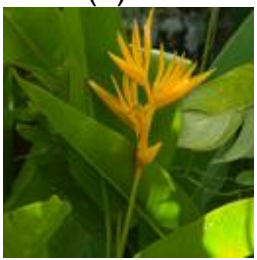

(f) Flower

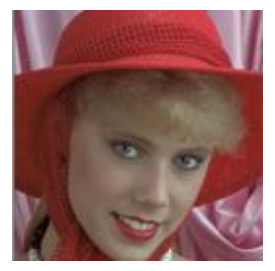

(e) Woman

Figure 3. Low Resolution Testing Images

In this experiment, we set the upscale factor to 2 and 4 , the patch size respectively to be $2 \times 2$ overlapped by 1 pixel and $4 \times 4$ overlapped by 2 pixels, FLANN nearest neighbor search of similar patches number to be 9 , number of pyramid layer is 2 and iterations number of IBP iterative back projection algorithm to be 20. Furthermore, in the local constraint algorithm we set distance threshold as 0.5 and $h$ as 10 . For color image super resolution reconstruction, because human vision is more sensitive to the luminance information than chrominance information, color images are converted from RGB to YIQ and the algorithm is applied to only Y Component. Then the rest of I, Q components are interpolated using in-built bicubic interpolation function in MATLAB.

To validate the effectiveness of our algorithm, the proposed algorithm is compared with some present algorithms: bicubic interpolation, sparse representation of Yang [8] and compressive sensing of Pan [15]. For each test, the quantitative measures named PSNR and SSIM were applied to evaluate the quality of the reconstructed image. Figure 4 shows the reconstruction results of "leaves" and "chest" image magnified by factor 2 . Figure 5 shows the "baby" image magnified by factor 4 (narrowed into half for the sake of comparisons and display). The comparisons of evaluations quality for reconstructed images are listed in Table 1. 


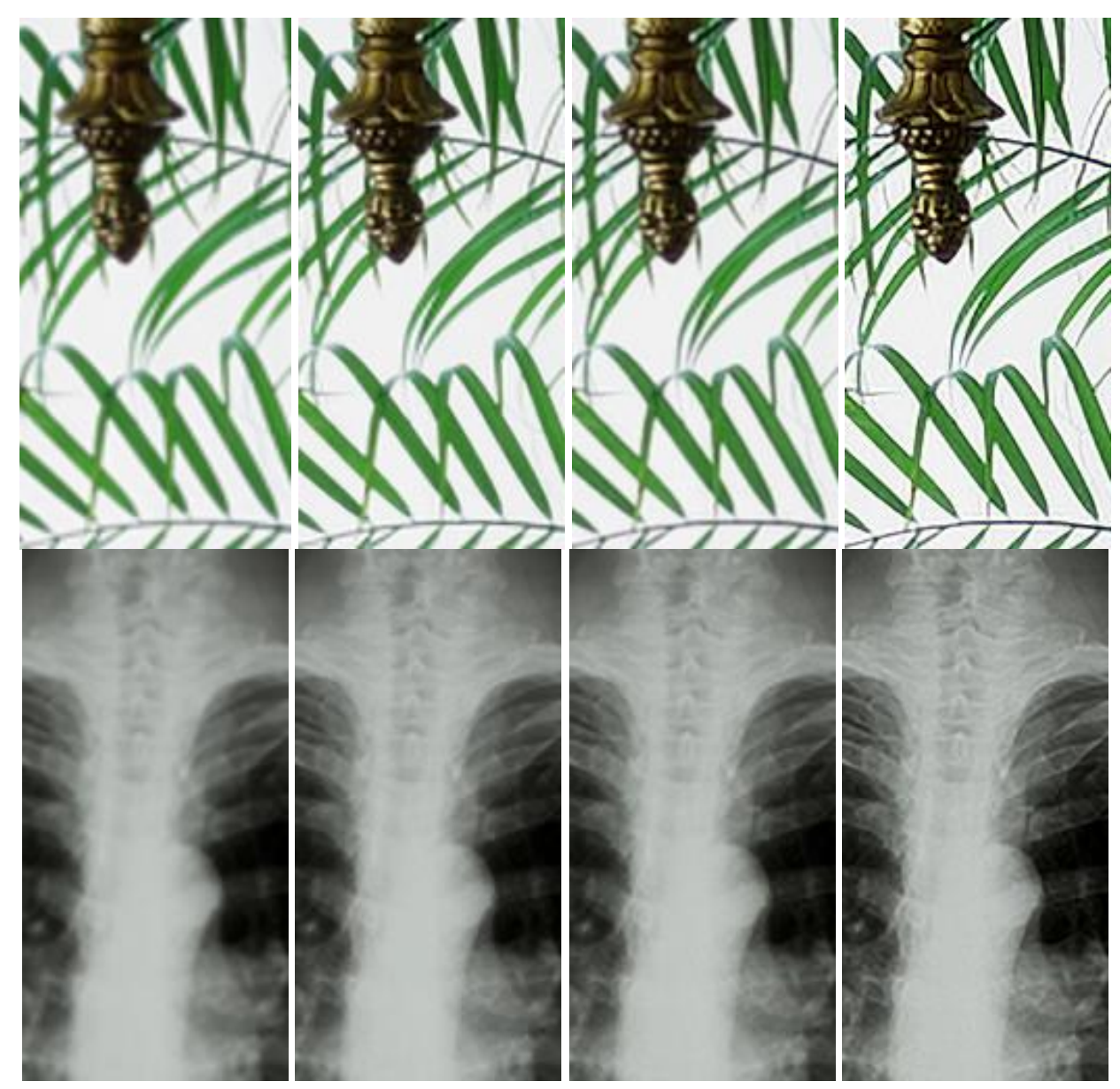

Figure 4. Comparisons of SR Result (x2) for "Leaves" and "Chest" Input Image from Left to Right: the Bicubic, Yang [8], Pan [15] and Our Proposed Method

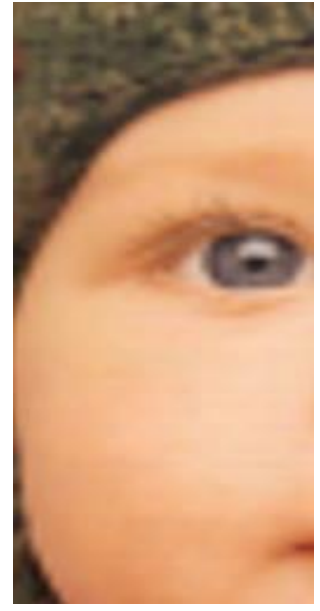

(a) Bicubic

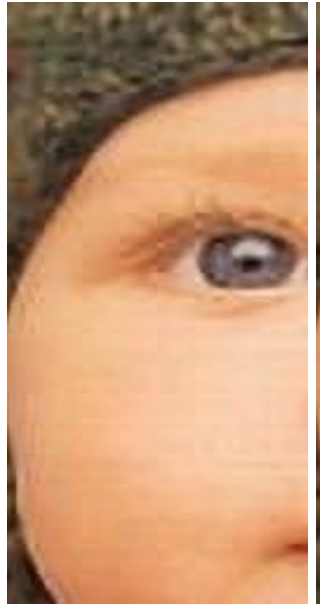

(b) Yang [8]

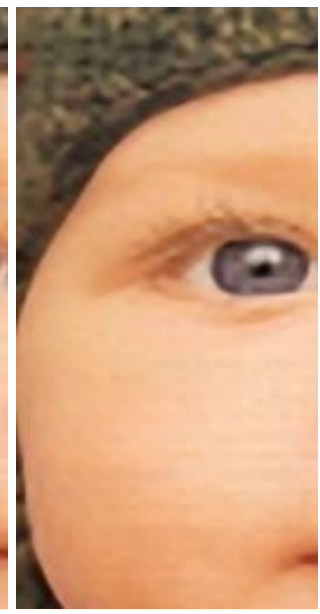

(c) Pan [15]

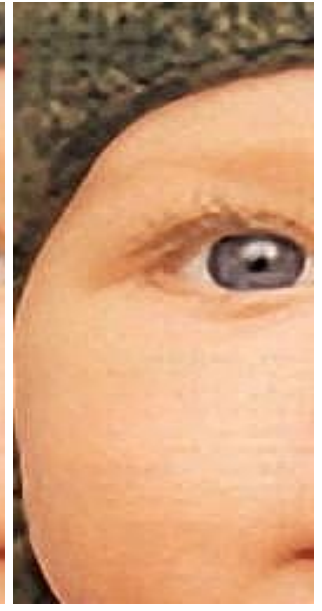

(d) Our Algorithm

Figure 5. Comparisons of SR Result (x4) for Baby Input Image 
Table 1. Image SR Performance Comparisons of PSNR (Left) and SSIM (Right)

\begin{tabular}{|c|c|c|c|c|c|}
\hline & $\begin{array}{c}\text { Upscale } \\
\text { factor }\end{array}$ & Bicubic & Yang[8] & Pan [15] & Proposed \\
\hline \multirow{3}{*}{ butterfly } & 2 & $26.268 / 0.916$ & $29.303 / 0.941$ & $29.435 / 0.953$ & $29.546 / 0.960$ \\
\cline { 2 - 6 } & 4 & $24.534 / 0.821$ & $25.403 / 0.849$ & $25.324 / 0.843$ & $25.474 / 0.847$ \\
\hline \multirow{3}{*}{ hat } & 2 & $30.089 / 0.871$ & $32.480 / 0.895$ & $32.628 / 0.914$ & $32.758 / 0.922$ \\
\cline { 2 - 6 } & 4 & $27.244 / 0.866$ & $28.325 / 0.872$ & $28.342 / 0.875$ & $28.422 / 0.879$ \\
\hline \multirow{3}{*}{ leaves } & 2 & $25.254 / 0.923$ & $27.435 / 0.932$ & $27.483 / 0.938$ & $27.644 / 0.951$ \\
\cline { 2 - 6 } & 4 & $23.246 / 0.867$ & $24.325 / 0.872$ & $24.364 / 0.876$ & $24.442 / 0.881$ \\
\hline \multirow{3}{*}{ parrots } & 2 & $30.132 / 0.930$ & $32.347 / 0.956$ & $32.336 / 0.950$ & $32.435 / 0.961$ \\
\cline { 2 - 6 } & 4 & $28.134 / 0.854$ & $29.245 / 0.864$ & 29.231 .0 .861 & $29.331 / 0.865$ \\
\hline \multirow{3}{*}{ woman } & 2 & $31.371 / 0.874$ & $33.424 / 0.931$ & $33.512 / 0.943$ & $33.632 / 0.952$ \\
\cline { 2 - 6 } & 4 & $28.533 / 0.832$ & $29.732 / 0.843$ & $29.641 / 0.841$ & $29.742 / 0.851$ \\
\hline \multirow{2}{*}{ chest } & 2 & $41.321 / 0.960$ & $43.157 / 0.971$ & $43.215 / 0.976$ & $43.464 / 0.981$ \\
\cline { 2 - 6 } & 4 & $39.173 / 0.884$ & $40.426 / 0.896$ & $40.452 / 0.901$ & $40.663 / 0.924$ \\
\hline \multirow{2}{*}{ baby } & 2 & $30.243 / 0.878$ & $32.183 / 0.894$ & $31.894 / 0.883$ & $32.021 / 0.908$ \\
\hline \multirow{2}{*}{ flower } & 4 & $28.375 / 0.865$ & $29.486 / 0.884$ & $29.464 / 0.881$ & $29.532 / 0.887$ \\
\cline { 2 - 6 } & 4 & $27.658 / 0.853$ & $30.247 / 0.891$ & $30.432 / 0.902$ & $30.421 / 0.896$ \\
\hline
\end{tabular}

\section{Conclusions}

In this paper, we presents a single image super-resolution reconstruction algorithm under the self-similarity and compressive sensing theory. Our proposed method omits the traditional external training database, avoiding the high dependency on the training database and the long-time training process. In order to predict more high-frequency details that low-resolution image need, we first rotate the low-resolution image by $90^{\circ}$, then use the medium-frequency patches to estimate the high-frequency patches. During the search and match process, the FLANN search method has been used to take place of the original ANN search strategy, which can improve the search speed. While obtaining the initial high-resolution image and dividing it into patches, we exploit them to generate the high-resolution dictionary by K-SVD, then perform the reconstruction process under the frame of compressive sensing theory. Experimental results show that the obtained training dictionary by our proposed algorithm is better, for it takes full advantage of the additional information implied in the image itself. When compared to other algorithms, our proposed algorithm shows the better performance, both in terms of objective metrics and subjective visual results.

\section{Acknowledgments}

This work is supported in part by the Key Scientific Research project Fund of Beijing Institute of Graphic Communication Ea201507 and Scientific Research Project of Beijing Municipal Education Commission KM201410015005. 


\section{References}

[1] R. Keys, "Cubic convolution interpolation for digital image processing", IEEE Trans. Acoust., Speech Signal Process, vol. 29, no. 6, (1981), pp. 1153-1160.

[2] S. Borman and R. L. Stevenson, "Super-resolution from Image Sequences-A Review", Proceedings of Midwest Symposium on Circuits and Systems. Washington D. C. USA: IEEE Press, vol. 30, no. 8, (1998), pp. 374-378.

[3] R. Tsai and T. Huang, "Multi-frame image restoration and registration", Advance in Computer Vision and Image Processing, vol. 1, no. 2, (1994), pp. 317-339.

[4] W. T. Freeman, T. R. Jones and E. C. Pasztor, "Example-based Super-resolution", Computer Graphics and Applications, vol. 22, no. 2, (2002), pp. 56-65.

[5] H. Chang, D. Y. Yeung and Xiong, "Super-resolution through neighbor embedding", CVPR 2004: Proceedings of the 2004 IEEE Computer Society Conference on Computer Vision and Pattern Recognition. Piscataway: IEEE Press, vol. 1, no. 1, (2004), pp. 275-282.

[6] J. Yang, J. Wright, and Y. Ma, "Image super-resolution as sparse representation of raw patches", IEEE Conf. Comput. Vis. Pattern Recognition, (2008), pp. 1-8.

[7] J. Yang, J. Wright and T. Huang, "Image super-resolution via sparse representation", IEEE Transactions on Image Processing, vol. 19, no. 1, (2010), pp. 2861-2873.

[8] J. Yang, Z. Wang, and T. Huang, "Coupled dictionary training for image super-resolution”, IEEE Trans. Image Process., vol. 21, no. 8, (2012), pp. 3467-3478.

[9] H. Zhang, Y. Zhang and T. Huang, "Efficient sparse representation based image super resolution via dual dictionary learning", in IEEE International Conference on Multimedia and Expo (ICME), Barcelona, Spain, vol. 57, no. 1, (2011), pp. 1-6.

[10] R. Zeyde, M. Elad and M. Protter, "On single image scale-up using sparse-representations", in $7^{\text {th }}$ International Conference on Curves and Surfaces, vol. 6920, (2010), pp. 711-730.

[11] M. Aharon, M. Elad and A. Bruckstein, "K-SVD: An algorithm for designing overcomplete dictionaries for sparse representation", IEEE Transactions on Signal Processing, vol. 54, no. 11, (2006), pp. 43114322.

[12] K. Zhang, X. Gao, D. Tao and X. Li, "Multi-scale dictionary for single image super-resolution", in IEEE Conference on Computer Vision and Pattern Recognition (CVPR), Providence, RI, (2012), pp. 11141121.

[13] J. Xu, C. Qi and Z. Chang, "Coupled K-SVD dictionary training for super-resolution", Image Processing (ICIP), 2014 IEEE International Conference on. IEEE, (2014), pp. 3910-3914.

[14] C. Y. Yang, J. B. Huang and M. H. Yang, "Exploiting self-similarities for single frame super-resolution. In: Proceedings of the 10thAsian conference on Computer vision", Berlin, Heidelberg: Springer, (2011), pp. 497-510.

[15] Z. Pan, J. Yu, H. Huang, S. Hu, A. Zhang, H. Ma and W. Sun, "Super resolution based on compressive sensing and structural self-similarity for remote sensing images", IEEE Transactions on Geoscience and Remote Sensing, vol. 51, no. 9, (2013), pp. 4864-4876.

[16] Z. Pan, H. Huang and W. Sun, "Super resolution of remote sensing image based on structure similarity in CS frame", Proceedings of SPIE - The International Society for Optical Engineering, vol. 8002, no. 1, pp. 554-561.

[17] S. Y. Yang, F. H. Sun, M. Wang, Z. Z. Lin and L. C. Jiao, "Novel Super Resolution Restoration of Remote Sensing Images Based on Compressive Sensing and Example Patches-Aided Dictionary Learning", 2011 International Workshop on Multi-Platform/Multi-Sensor Remote Sensing and Mapping (M2RSM), (2011), pp. 1-6.

[18] Z. Zhu, F. Guo and H. Yu, "Fast Single Image Super-Resolution via Self-Example Learning and Sparse Representation", IEEE Transactions on Multimedia, vol. 16, no. 8, (2014), pp. 2178-2190.

[19] D. Glasner, S. Bagon and M. Irani, "Super-resolution from a single image", in IEEE International Conference on Computer Vision (ICCV), Kyoto, Japan, (2009), pp. 349-356.

[20] S.-J. Park, O.-Y. Lee and J.-O. Kim, "Self-similarity based image super-resolution on frequency domain", proc. of APSIPA ASC 2013, (2013).

[21] G. Davis, S. Mallat and M. Avellaneda, "Adaptive greedy approximation", Constructive Approximation, vol. 13, no. 1, (1997), pp. 57-98.

[22] S. S. Chen, D. L. Donoho and M. A. Saunders, "Atomic decomposition by basispursuits", SIAM Journal on Scientific Computing, vol. 20, (1999), pp. 33-61.

[23] J. Tropp and A. Gilbert, "Signal Recovery from Random Measurements via Orthogonal Matching Pursuit", IEEE Trans. Info. Theory (S0018-9448), vol. 53, no. 12, (2007), pp. 4655-4666.

[24] A. Buades, B. Coll and J. M. Morel, "Non-local Image and Movie Denoising", International Journal of Computer Vision (S0920-5691), vol. 76, no. 2, (2008), pp. 123-139.

[25] R. Tibshirani, "Regression Shrinkage and Selection via the Lasso", Journal of the Royal Statistical Society Series B (S0035-9246), vol. 58, no. 1, (1996), pp. 267-288. 


\section{Authors}

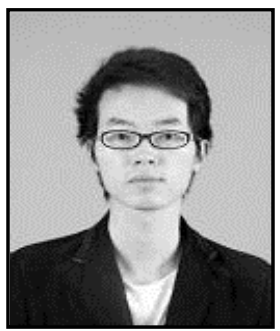

Luyang Yao, He received his Master's degree in Information Engineering from Beijing Institute of Graphic Communication, China in 2014, where his research interests include Graphic image processing, pattern recognition and computer vision.

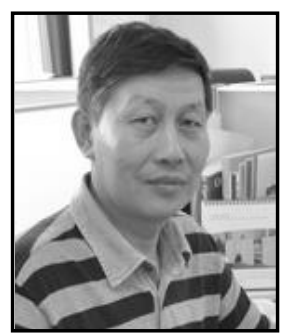

Kai Xie, He received the M.S. degree from the North Jiaotong University in 1987 and the Ph.D. degree from the School of Computer Science and Technology at Harbin Institute of Technology in 2005. He is now a Professor of Beijing Institute of Graphic and Communication. His research interests include digital signal and image processing, convex optimization, machine learning and information theory. 
International Journal of Signal Processing, Image Processing and Pattern Recognition Vol. 10, No. 4 (2017) 